Editorial

\title{
Quantitative Methods for Molecular Diagnostic and Therapeutic Imaging
}

\author{
Quanzheng $\mathrm{Li}^{凶}$ \\ Center for Advanced Medical Imaging Sciences, Harvard Medical School, Massachusetts General Hospital, Boston, MA, USA
}

$\square$ Corresponding author: Quanzheng Li, 55 Fruit St, White 427, Boston MA 02114, USA. Tel: 1(617)643-9481, Fax: 1(617)726-6165, Email: Li.Quanzheng@mgh.harvard.edu

( ) Ivyspring International Publisher. This is an open-access article distributed under the terms of the Creative Commons License (http://creativecommons.org/ licenses/by-nc-nd/3.0/). Reproduction is permitted for personal, noncommercial use, provided that the article is in whole, unmodified, and properly cited.

Received: 2013.08.20; Accepted: 2013.08.20; Published: 2013.09.19

\begin{abstract}
This theme issue provides an overview on the basic quantitative methods, an in-depth discussion on the cutting-edge quantitative analysis approaches as well as their applications for both static and dynamic molecular diagnostic and therapeutic imaging.
\end{abstract}

Key words: tomography, image quality metrics, local impulse response, resolution, variance.

The field of molecular imaging has progressed from qualitative imaging to semi-quantitative and then quantitative imaging. Compared to qualitative imaging, quantitative imaging can provide not only more accurate and task-specific information for various diagnostic applications, but also more precise evaluation and more appropriate management of treatment. Furthermore, once a quantitative measure of diagnosis or treatment is available, one can optimize the performance of diagnosis or treatment by adapting the parameters in the mathematical description of the quantitative measurement.

Because quantitative methods mathematically describe both diagnostic and therapeutic molecular imaging, they effectively provide a bridge to naturally merge these two components together into a new unified quantitative mathematical framework for the analysis and optimization of theranostics. We therefore believe quantitative methods will play an important role in the development of theranostics and take the initiative to publish this special issue on quantitative imaging, in which 6 groups of researchers are invited to share their experience, expertise, ideas and results on quantitative molecular imaging.

The evolution of methodology for the analysis of
Positron Emission Tomography (PET) data is a perfect example to demonstrate the transition from qualitative to quantitative imaging. Soon after people applied a semi-quantitative standardized update value (SUV) to replace the visual evaluation of the image quality, they realized that SUV has a lot of disadvantages (e.g. inconsistency). A number of modified SUV methods as well as quantitative analysis methods are then developed to overcome the deficiencies of SUV. Bai et al. reviewed SUV and its derivatives and the factors that impede semi-quantitative assessment and limit its accuracy and reproducibility, with special emphasis on SUV analysis [1]. They also summarized the current efforts to improve the accuracy of tumor uptake measurements, characterize overall metabolic tumor burden and heterogeneity of tumor uptake, and account for the effects of image noise.

In spite of the diversity of imaging modalities and applications, the underlying methodologies for quantitative imaging are the same. For static images, these include the analysis and optimization of image quality in terms of variance, resolution, contrast recovery and lesion detectability. Dutta et al. review statistical analysis techniques that enable us to com- 
pute the properties of static molecular images [2]. In addition, these also allow us to control and enhance imaging performance. The practical applications where performance improvement is achieved by applying these ideas to the contexts of both hardware and image reconstruction are also reviewed. While most of the discussed techniques were developed for emission tomography, the general methods are extensible to other imaging modalities as well.

For a specific diagnostic or therapeutic task, human and computer observers are usually applied to study the sensitivity-specificity trade-off. He and Park thoroughly discuss the basic concepts of model observers, which include the mathematical foundations and psychophysical considerations in designing both optimal observers for optimizing imaging systems and anthropomorphic observers for modeling human observers, as well as a few state-of-the-art computational techniques for estimating model observers and the principles of implementing these techniques [3]. A few applications of model observers in medical imaging research are also reviewed.

The fundamental methodology used for dynamic quantitative imaging is compartment modeling. This special issue introduces two cutting-edge techniques in kinetic modeling. For a more basic introduction to kinetic analysis, we recommend several excellent reviews by other authors on this topic, such as [4-6]. Wang and Qi review recent progress on direct estimation of parametric images of both linear and nonlinear kinetic models using spatiotemporal information in raw projection data [7]. These direct estimation algorithms can include accurate noise modeling and therefore achieve better image quality than conventional indirect methods. Different PET tracers can capture different aspects of the physiology of diseases; techniques that can rapidly image multiple tracers in a single scan could potentially provide additional critical information compared to traditional dynamic single tracer scans. In [8], Kadrmas and Hoffman summarize past and ongoing work in multi-tracer PET tumor imaging, and then organize and describe the main algorithmic approaches for achieving multi-tracer PET signal-recovery. In these approaches, dynamic imaging is generally used and signal-recovery processing is applied.

The above quantitative analysis methods have been applied to different preclinical and clinical applications using different modalities, such as PET, Single Photon Emission Computed Tomography (SPECT) and optical imaging. As an example, an application of quantitative imaging in proton therapy is given in [9], in which Zhu et al. review the current status of proton therapy verification with PET imag- ing. Proton therapy is very sensitive to uncertainties introduced during treatment planning and dose delivery. PET imaging can detect the distribution of proton-induced positron emitters and is the only practical approach for in vivo, in situ verification of proton therapy. The different data detecting systems (in-beam, in-room and off-line PET), calculation methods for the prediction of proton-induced PET activity distributions, and approaches for data evaluation are discussed in [9].

Quantitative analysis is now a very productive and active research front in molecular imaging. We anticipate the introduction of quantitative methods to theranostics will produce an emerging, rapid growing and fruitful research field, which in turn will advance the development of theranostics. We hope this special issue can serve as an overview of the previous methods as well as a starting point toward this new exciting direction.

\section{Competing Interests}

The authors have declared that no competing interest exists.

\section{References}

1. Bai B, Bading J, Conti PS. Tumor Quantification in Clinical Positron Emission Tomography. Theranostics 2013; 3(10):787-801.

2. Dutta J, Ahn S, Li Q. Quantitative Statistical Methods for Image Quality Assessment. Theranostics 2013;3(10):741-756.

3. He X, Park S. Model Observers in Medical Imaging Research. Theranostics 2013; 3(10):774-786.

4. Carson RE. Tracer kinetic modeling in PET. In: Bailey DL, Townsend DW, Valk PE, Maisey MN, eds. Positron Emission Tomography: Basic Sciences. Springer, 2005: 127-159.

5. Morris, ED, Christopher JE, Kathleen CS, Bradley TC, Raymond FMJr, and Ronald EF. Kinetic modeling in positron emission tomography. In: Wernick MN and Aarsvold JN, eds. Emission Tomography: The Fundamentals of PET and SPECT. Academic Press, 2004: 499-540.

6. Zaidi H. Tracer kinetic modeling in PET. PET Clinics 2007;2: 267-77.

7. Wang G, Qi J. Direct Estimation of Kinetic Parametric Images for Dynamic PET. Theranostics 2013; 3(10):802-815.

8. Kadrmas DJ, Hoffman JM. Methodology for Quantitative Rapid Multi-Tracer PET Tumor Characterizations. Theranostics 2013; 3(10):757-773.

9. Zhu $X$, Fakhri GE. Proton Therapy Verification with PET Imaging. Theranostics 2013;3(10):731-740. 